\title{
Control of Active Microvibration Isolation Equipment by Consideration of the Air Pressure Characteristics in a Tube*
}

\author{
Sachio MAEKAWA ${ }^{* *}$, Masaomi WADA**, Yasutaka TAGAWA**, \\ Shizuo IMAOKA $^{* *}$ and Masaki HAYATSU** \\ **Hitachi Plant Technologies, Ltd. \\ 537 Kami-hongo, Matsudo-shi, Chiba, 271-0064, Japan \\ E-mail: sachio.maekawa.xe@hitachi-pt.com \\ ***Tokyo University of Agriculture and Technology \\ 2-24-16 Naka-cho, Koganei-shi, Tokyo, 184-8588, Japan \\ E-mail: tagawa@cc.tuat.ac.jp
}

\begin{abstract}
The range of applications using microvibration isolation equipment is being extended to devices for which the influence of vibration had not previously been considered. As a representative example, the application of active microvibration isolation equipment using an air spring actuator, suitable for MRI and NMR that operate in a strong magnetic field, is examined. In order to be used in a strong magnetic field, the active microvibration isolation equipment needs to have an actuator made from a nonmagnetic material. The servo valve that controls the air pressure in the actuator, however, must be installed away from the strong magnetic field since it cannot be fabricated from a nonmagnetic material. This necessitates the use of a long air supply tube to connect the servo valve to the actuator. Since this long tube affects the actuator characteristics, adequate vibration isolation performance cannot be attained with a controller designed using the conventional equipment model. In this study, a new equipment model is proposed by analyzing the characteristics of the active microvibration isolation equipment, and the control system is designed for the model using the model matching technique. The equipment model and control technique are validated by simulation and experiment.
\end{abstract}

Key words: Vibration Isolation, Vibration Control Device, Air Pressure Characteristics, Vibration Coupled with Fluid Motion, Modeling, Model Matching

\section{Introduction}

Recent improvements in the accuracy of precision devices have led to stricter requirements for vibration isolation. In addition, the range of applications using vibration isolation equipments is being extended to devices for which the influence of vibration had not previously been considered. Typical examples of such devices are magnetic resonance imaging (MRI) devices and nuclear magnetic resonance (NMR) analyzers that leak strong magnetic fields. Currently, these devices are generally isolated from vibrations using a passive method such as an air spring, but an active method, which offers improved vibration isolation, may be applied in the future.

Actuators for active vibration isolation systems that are under investigation include piezoelectric actuators, magnetostrictors, linear actuators, and air actuators. Currently, 
various studies ${ }^{(1) \sim(3)}$ are being done into enhancing the vibration isolation performance of these systems. This study focuses on a vibration isolation system using an air actuator that has a long stroke and facilitates attitude control, while ensuring good vibration isolation performance.

Equipment containing magnetic materials cannot be installed in the vicinity of MRI or NMR system because they may affect the accuracy of measurement. In addition, there is also the problem that leakage of a strong magnetic field from the MRI or NMR system may cause a device made from magnetic materials to malfunction. The main body of an air actuator may be made from non-magnetic materials but the servo valve supplying air to the actuator may not. In the conventional system, the servo valve is directly installed in an air actuator. However, in the new system developed for MRI or NMR, it must be kept away from strong magnetic fields to prevent malfunctions from occurring. To ensure a sufficient distance, a long tube is needed between the actuator and the servo valve. However, the actuator characteristics are found to be dependent on the properties of the tube $e^{(4),(5)}$.

In this study, we create a new model by analyzing the characteristics of an active vibration isolation system having a tube sufficiently long to affect the characteristics of the system, we design a controller based on this model, and verify its effectiveness by simulation and experiment.

\section{Experimental Setup}

Figures 1 and 2 show the configuration of the experimental setup used in this study. The system consists of a vibration isolation table, roll seal type air actuators, a support table, acceleration sensors, a personal computer, and a digital signal processor (DSP). The vibration isolation table, having dimensions of $1.2 \times 1.2 \times 0.2 \mathrm{~m}$ and a mass of $860 \mathrm{~kg}$, is installed on the support table using the air actuators. The four air actuators are installed in the vertical and horizontal directions. To avoid the influence of a magnetic field, the air supply servo valve, which is usually attached directly to the actuator, is installed away from the actuator. Then a nylon tube (inner diameter: $8 \mathrm{~mm}$ ) is used to connect the actuator and the servo valve. The air pressure supplied to the actuator is set to $0.5 \mathrm{MPa}$. For this study, $5-\mathrm{m}$ and $20-\mathrm{m}$ tubes are prepared. The vibration isolation table has three acceleration sensors in the vertical direction and three in the horizontal direction. An acceleration sensor is installed on the installation floor. To study the enhancement of vibration isolation performance in the vertical direction, only the actuators and acceleration sensors in the vertical direction are used. For active control investigated in this study, the DSP captures the electric signals from the acceleration sensors, computes the operating voltage for the servo valve in order to control each actuator, and inputs the voltage to each servo valve to drive the corresponding actuator.

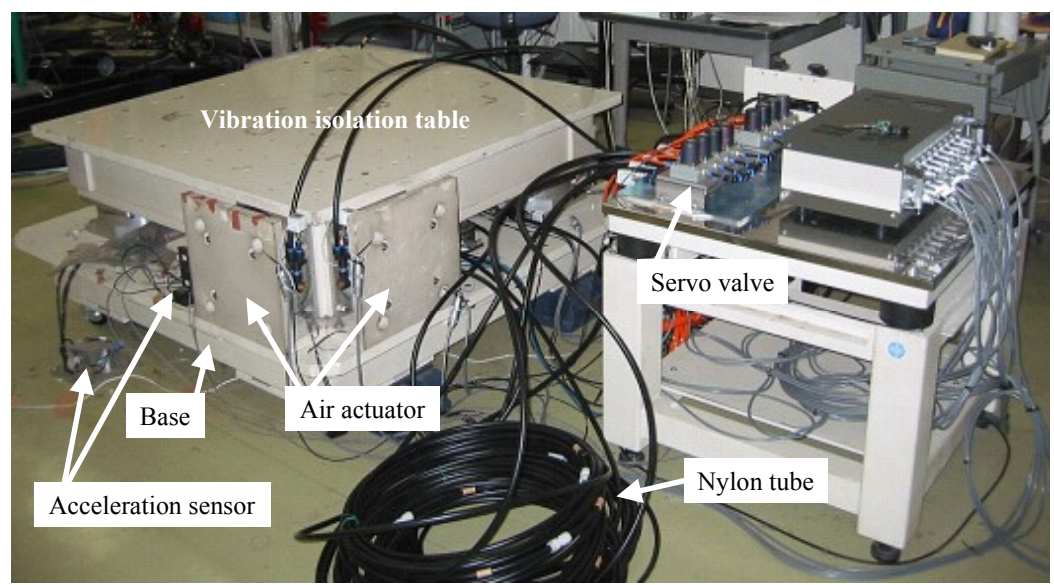

Fig. 1 Photograph of the experimental setup 


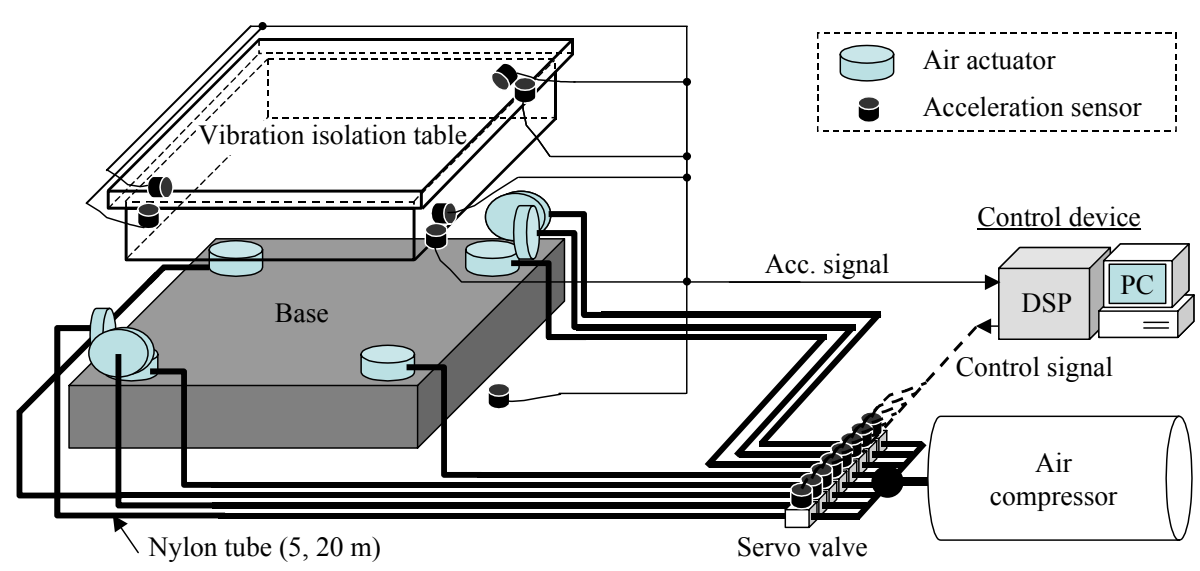

Fig. 2 Schematic view of the experimental setup

The system also has displacement sensors to control the displacement of the actuators by PI control. This control is performed at a frequency lower than the active control frequency used in this study.

\section{Modeling}

\subsection{System Characteristics Check}

To investigate the influence of the nylon tube on the system characteristics, the transfer characteristics from the floor acceleration to the vibration isolation table acceleration and those of the vibration isolation table acceleration to the actuator operating voltage were measured. For the former, the acceleration of the floor and the acceleration of the vibration isolation table were measured. For the latter, the acceleration on the vibration isolation table was measured when a sine-wave sweep voltage of 0.1 to $100 \mathrm{~Hz}$ was applied to the air actuator. Figure 3 shows the experimental results.

In Fig.3(a), the first resonance frequency appears at about $10 \mathrm{~Hz}$ and there are no other resonance peaks when the tube is not installed. However, when 5-m tubes are installed, the first resonance frequency occurs at about $6 \mathrm{~Hz}$, while it occurs at about $3 \mathrm{~Hz}$ when $20-\mathrm{m}$ tubes are installed. In both cases, many resonance peaks appear in the frequency range between the first resonance frequency and $100 \mathrm{~Hz}$. As the length of the tube is increased, the resonance frequencies shift to a lower frequency and resonance and anti-resonance peaks occur alternately. The phase is delayed by 180 degrees at the resonance peak and 180 degrees in advance at the anti-resonance peak. Therefore, the phase is not delayed by more than 180 degrees.
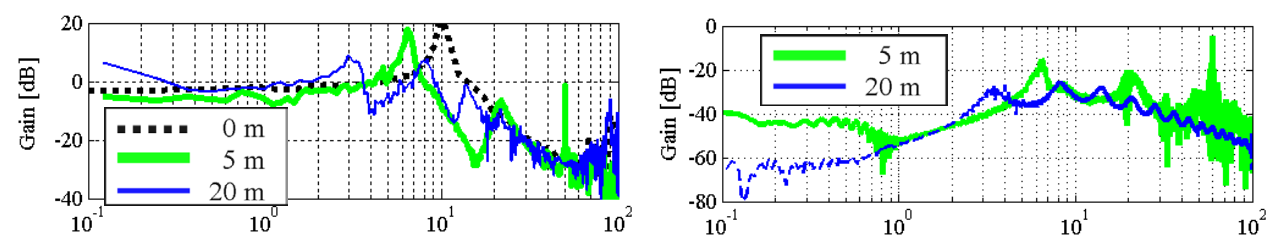

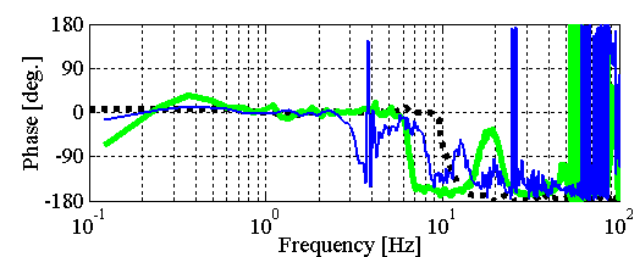

(a) From floor acc. to table acc.

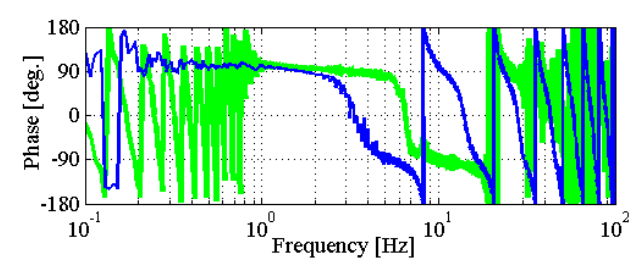

(b) From input volt. to table acc.

Fig. 3 Frequency transmissibility characteristics 
The result in Fig.3(b) shows similar characteristics to the one in Fig.3(a). As the length of the tube increases, the number of resonance peaks increases. But the phase is delayed by 180 degrees at each resonance peak and there are no anti-resonance peaks.

These results show that the tube connection delays the air supply to the actuators after valve opening and closing.

\subsection{System Model}

In $\S 3.1$, it was found that the tube connection greatly modifies the system characteristics. Figure 4 shows a system model based on these results. In a rigid model of the vibration isolation table, the system model has virtual mass points connected in series where the air in the tube is regarded as virtual mass. In Fig.4, $m_{1}, c_{1}, k_{l}, z_{1}$, and $z_{0}$ represent the mass, damping coefficient, spring constant, relative displacement of the vibration isolation table and the floor displacement, respectively. Treating the air in the tube as a virtual mass, $m_{i}, c_{i}, k_{i}$, and $z_{i}$ (where $i=2$ to $n$ ) are defined to be the virtual mass, damping coefficient, spring constant, and displacement of the virtual mass, respectively.

From this model, the equation of motion at each mass point becomes the following:

$$
\left.\left.\left[M_{n}\right]\right\} \begin{array}{c}
\ddot{z}_{1}+\ddot{z}_{0} \\
\ddot{z}_{2}+\ddot{z}_{0} \\
\vdots \\
\ddot{z}_{n}+\ddot{z}_{0}
\end{array}\right\}+\left[C_{n}\right]\left\{\begin{array}{c}
\dot{z}_{1} \\
\dot{z}_{2} \\
\vdots \\
\dot{z}_{n}
\end{array}\right\}+\left[K_{n}\right]\left\{\begin{array}{c}
z_{1} \\
z_{2} \\
\vdots \\
z_{n}
\end{array}\right\}=\left[H_{n}\right] f
$$

where, $\left[M_{n}\right],\left[C_{n}\right]$, and $\left[K_{n}\right]$ are the mass matrix, damping matrix, and stiffness matrix respectively, having $n$ rows and $n$ columns and $\left[H_{n}\right]$ represents $\left[\begin{array}{llll}0 & \cdots & 0 & 1\end{array}\right]^{T}$ which has $n$ rows and one column.

Equation (1) couples the displacement at each mass point. To simplify the system, modal analysis is conducted and a non-coupled equation of motion is derived for each vibration mode. If the $n$-th modal coordinate vector $\left\{\xi_{n}\right\}\left(\left\{z_{n}\right\}=\left[\phi_{n}\right]\left\{\xi_{n}\right\}\right)$ is introduced and the damping matrix is assumed to be a linear sum of the mass and stiffness matrices, Eq.(1) becomes the expression shown below and the system model can be expressed as the $n$-th equation of motion non-coupled for each vibration mode:

$$
\left.\left\{\ddot{\xi_{n}}\right\}+\left[2 \zeta_{n} \omega_{n}\right]\left\{\dot{\xi}_{n}\right\}+\left[\omega_{n}^{2}\right]\left\{\xi_{n}\right\}=\left[\phi_{n}\right]^{T}\left[H_{n}\right] f-\left[\phi_{n}\right]^{T}\left[M_{n}\right]\right]\left(\ddot{z}_{0}\right\}
$$

where, $\left[2 \zeta_{n} \omega_{n}\right]$ and $\left[\omega_{n}{ }^{2}\right]$ are $n$ by $n$ diagonal matrices that have the elements $2 \zeta_{i} \omega_{i}$ and $\omega_{i}{ }^{2}$ at row $i$ and column $i$, respectively. [ $\left.\phi_{n}\right]$ is the normalized modal matrix of $n$ rows and $n$ columns where $\zeta_{i}$ and $\omega_{i}$ represent the $i$-th damping ratio and natural angular frequency, respectively.

If the relationship between the actuator generated force $f$ and the actuator operating voltage $u$ is assumed to be the first order lag system, the following equation can be derived:

$$
\dot{f}=-\frac{1}{T} f+\frac{L}{T} u
$$

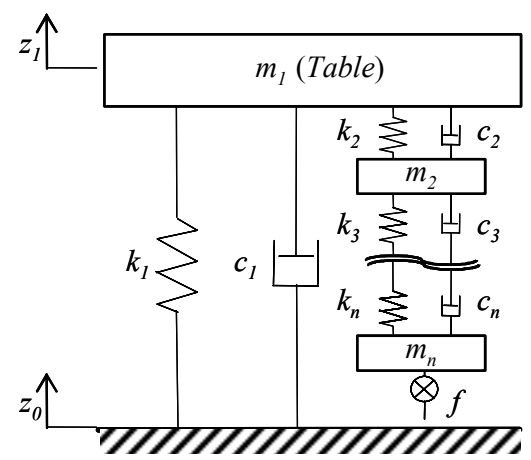

$m_{1}:$ Table mass

$m_{i}$ : Virtual mass $(i=2$ to $n)$

$k_{i}$ : Spring constant $(i=1$ to $n)$

$c_{i}$ : Damping coefficient $(i=1$ to $n)$

$z_{0}:$ Floor displacement

$z_{i}$ : Relative displacement for floor displacement $(i=1$ to $n)$

$f:$ Actuator generated force

Fig. 4 Analytical model 
where, $T$ represents the time constant and $L$ represents the actuator gain. From Eqs.(2) and (3), the following state equation can be obtained:

$$
\left\{\begin{array}{c}
\dot{\xi}_{1} \\
\vdots \\
\xi_{n} \\
\ddot{\xi_{1}} \\
\vdots \\
\ddot{\xi_{n}} \\
\dot{f}
\end{array}\right\}=\left[\begin{array}{ccc}
O(n \times n) & \boldsymbol{I}(n \times n) & O(n \times 1) \\
-\left[\omega_{n}^{2}\right] & -\left[2 \zeta_{n} \omega_{n}\right] & \phi_{n 1} \\
& & \phi_{n n} \\
O(1 \times n) & O(1 \times n) & -\frac{1}{T}
\end{array}\right]\left[\begin{array}{c}
\xi_{1} \\
\vdots \\
\xi_{n} \\
\dot{\xi_{1}} \\
\vdots \\
\dot{\xi}_{n} \\
f
\end{array}\right]+\left[\begin{array}{c}
O(2 n \times 1) \\
\frac{L}{T}
\end{array}\right] u-\left[\begin{array}{c}
O(n \times 1) \\
\overline{\phi_{1}} \\
\vdots \\
\overline{\phi_{n n}} \\
0
\end{array}\right] \ddot{z}_{0}
$$

where, $\boldsymbol{O}(i \times j)$ represents the zero matrix having $i$ rows and $j$ columns, and $\boldsymbol{I}(i \times i)$ represents the unit matrix having $i$ rows and $i$ columns, $\phi_{i j}$ represents the element at row $i$ and column $j$ of the normalized modal matrix; then $m_{i} \phi_{i i}$ replaces $\overline{\phi_{i i}}$.

The acceleration on the vibration isolation table can be expressed using the modal coordinate vectors as follows:

$$
\ddot{z}_{1}=\phi_{11} \ddot{\xi}_{1}+\phi_{12} \ddot{\xi}_{2}+\cdots+\phi_{1 n} \ddot{\xi}_{n}
$$

Since the output $Y$ is the absolute acceleration $\ddot{z}_{1}+\ddot{z}_{0}$ of the vibration isolation table, Eq.(4) can be transformed into the following output equation.

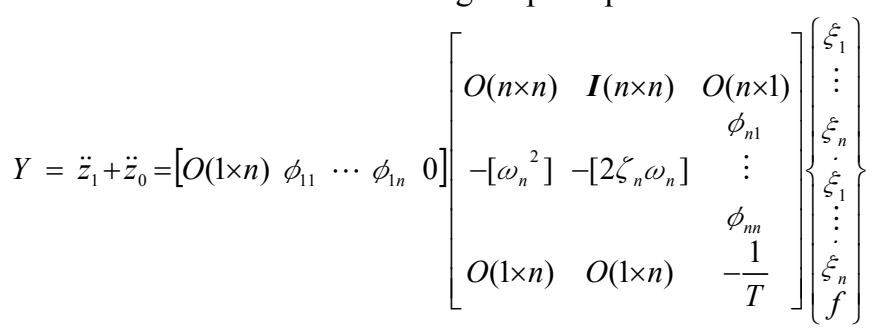

For identifying the system, $\zeta_{i}$ and $\omega_{i}$ in each mode and the modal coefficient $\phi_{i j}$ and $\overline{\phi_{i}}$ in Eqs.(4) and (6) are adjusted on the basis of the results obtained in $\S 3.1$.

\subsection{Simulation of Identification}

Table 1 indicates the identified parameters for tube length of 5 and $20 \mathrm{~m}$ and Figs. 5 and 6 show the identification results. For parameters not shown in Table 1, $T$ and $L$ are set to 1 and 50, respectively. The system model in Fig. 4 depicted a system having $n-1$ virtual masses. To simplify the model, we identified a third order system model by adding two air virtual masses. The experimental results almost match the frequency transmissibility characteristics identified from the transfer functions, confirming the validity of the model. While a model having three resonance peaks was considered this time, it can be extended for higher order systems by increasing the number of virtual masses of the model.

Table 1 Identified parameters

\begin{tabular}{c|c|c|c|c|c|c}
\hline \hline \multirow{2}{*}{$\begin{array}{c}\text { Tube length } \\
{[\mathrm{m}]}\end{array}$} & \multirow{2}{*}{$\begin{array}{c}\text { Order } \\
\end{array}$} & \multicolumn{6}{|c}{ Parameters $(i=1$ to 3$)$} \\
\cline { 3 - 7 } & 1 & $\omega_{i}$ & $\zeta_{i}$ & $\phi_{1 i}$ & $\phi_{3 i}$ & $\overline{\phi_{i i}}$ \\
\hline \multirow{3}{*}{5} & 2 & 133 & 0.042 & 1.0 & -0.018 & 0.04 \\
\cline { 2 - 7 } & 3 & 314 & 0.042 & 1.0 & 0.015 & 0.005 \\
\hline \multirow{3}{*}{20} & 1 & 20.7 & 0.115 & 1.0 & 0.004 & 0.5 \\
\cline { 2 - 7 } & 2 & 51.5 & 0.118 & 1.0 & -0.011 & 0.5 \\
\cline { 2 - 7 } & 3 & 88.0 & 0.08 & 1.0 & 0.012 & 0.09 \\
\hline
\end{tabular}



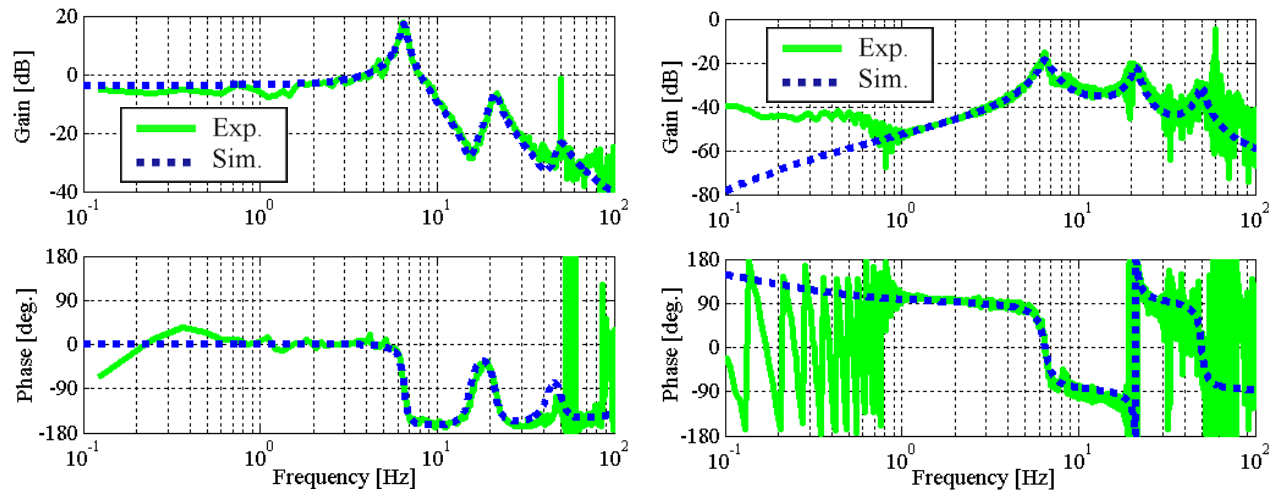

(a) From floor acc. to table acc.

(b) From input volt. to table acc.

Fig. 5 Identification results for transmissibility characteristics (tube length: $5 \mathrm{~m}$ )
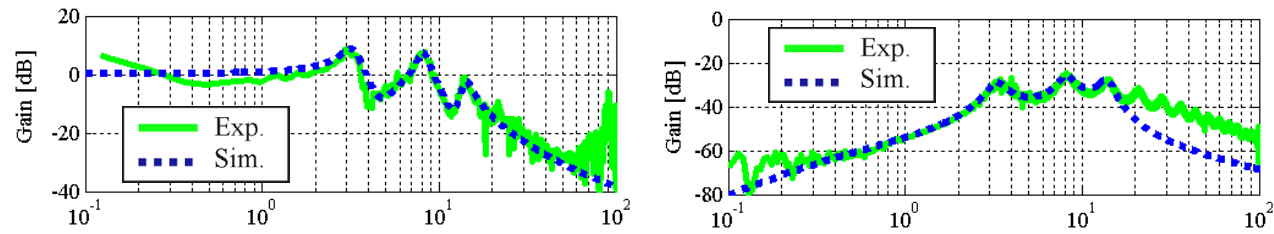

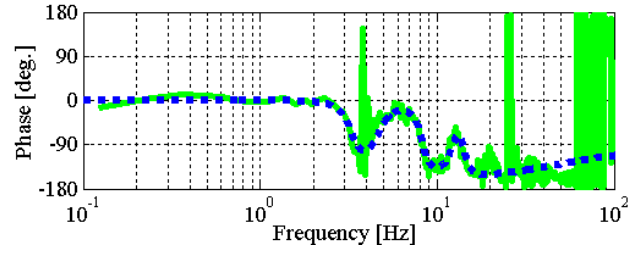

(a) From floor acc. to table acc.

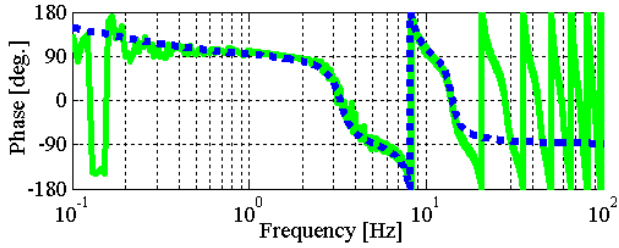

(b) From input volt. to table acc.

Fig. 6 Identification results for transmissibility characteristics (tube length: $20 \mathrm{~m}$ )

\section{Model Matching Technique}

To realize good performance of vibration isolation, the model matching technique ${ }^{(6),(7)}$ for controller design is adopted in this study. In this technique, a closed-loop transfer function (the closed-loop transfer function $W_{d y}$ from the floor acceleration to the acceleration of the vibration isolation table in this study) is predefined in order to attain a desired characteristic. Then, using this transfer function, a controller is derived to realize it. Figure 7 shows a block diagram of the control system; in this figure, $P_{u y}$ and $P_{d y}$ represent the open-loop transfer functions from the actuator operating voltage $u$ to the acceleration on the vibration isolation table $y$ and from the floor acceleration $d$ to the acceleration of the vibration isolation table $y$ and $C_{y u}$ represents the controller.

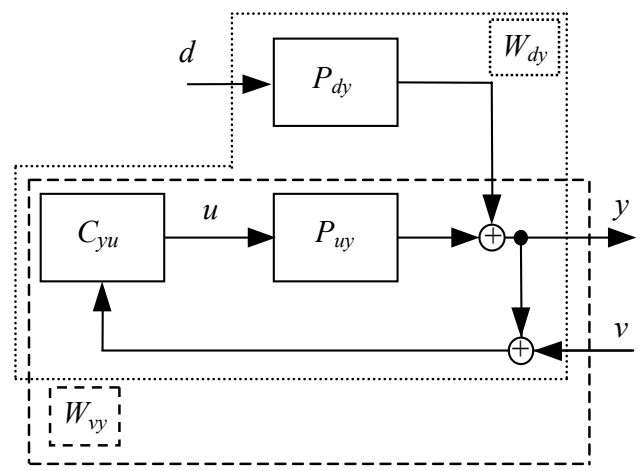

$u$ : Input (actuator operating voltage)

$y$ : Output (table acceleration)

$v$ : Noise

$d$ : Disturbance (floor acceleration)

$P_{d y}:$ Open-loop transfer function (from $d$ to $y$ )

$P_{u y}$ : Open-loop transfer function (from $u$ to $y$ )

$W_{d y}$ : Closed-loop transfer function (from $d$ to $y$ )

$W_{v y}$ : Closed-loop transfer function (from $v$ to $y$ )

$C_{y u}$ : Feedback controller

Fig. 7 Block diagram of the control system 
From the block diagram, the closed-loop transfer function $W_{d y}$ from the floor acceleration $d$ to the acceleration of the vibration isolation table $y$ and the closed-loop transfer function $W_{v y}$ from the observed noise $v$ to the acceleration of the vibration isolation table $y$ become:

$$
\begin{aligned}
& W_{d y}=P_{d y}\left(1-P_{u y} C_{y u}\right)^{-1} \\
& W_{v y}=P_{u y} C_{y u}\left(1-P_{u y} C_{y u}\right)^{-1}
\end{aligned}
$$

Equation (8) is transformed to the following:

$$
1+W_{v y}=\left(1-P_{u y} C_{y u}\right)^{-1}
$$

Using Eq.(7) Eq.(9), $C_{y u}$ and $W_{d y}$ can be expressed as follows:

$$
\begin{aligned}
& C_{y u}=W_{v y} P_{u y}^{-1}\left(1+W_{v y}\right)^{-1} \\
& W_{d y}=P_{d y}\left(1+W_{v y}\right)
\end{aligned}
$$

Using the above equations, first of all, $W_{d y}$ is set to the desired characteristic. Then $W_{v y}$ is obtained using Eq.(11), and Eq.(10) is used to determine $C_{y u}$.

The transfer functions $P_{u y}$ and $P_{d y}$ are matched to the model having two resonance peaks to reduce the number of degrees of the controller for mounting on the system. In this study, $W_{d y}$ is set as follows:

$$
W_{d y}=\frac{\left(s+T^{-1}\right)\left(\alpha_{3} s^{3}+\cdots+\alpha_{0}\right)\left(s^{6}+\gamma_{5} s^{5}+\cdots+\gamma_{0}\right)}{\left(s-p_{1}\right)\left(s-p_{2}\right) \cdots\left(s-p_{11}\right)}
$$

where, $\alpha_{i}$ represents the coefficient of the molecular term of $P_{d y}$, and $\gamma_{i}$ and $p_{i}$ represent the coefficient and the pole, respectively. According to the design guidelines of the control system, in order to attenuate the first and second resonance peaks at $W_{d y}$, four poles should be arranged for each peak and one actuator pole is set. Then two poles are arranged so that non-modeled resonance peaks in the high frequency range will not adversely affect the control system.

\section{Vibration Isolation Performance}

\subsection{Simulation Results}

The frequency transmissibility characteristics from the floor acceleration to the acceleration of the vibration isolation table were analyzed by using the numerical analysis software 'MATLAB' to design a controller having excellent vibration isolation performance.

Considering the application to an actual system, the controller was designed for a system model that included up to the second resonance frequency of $P_{u y}$ to lower the degree of the controller. Then the designed controller to a system model that included up to the third resonance frequency was applied. Figure 8 shows the simulation results.
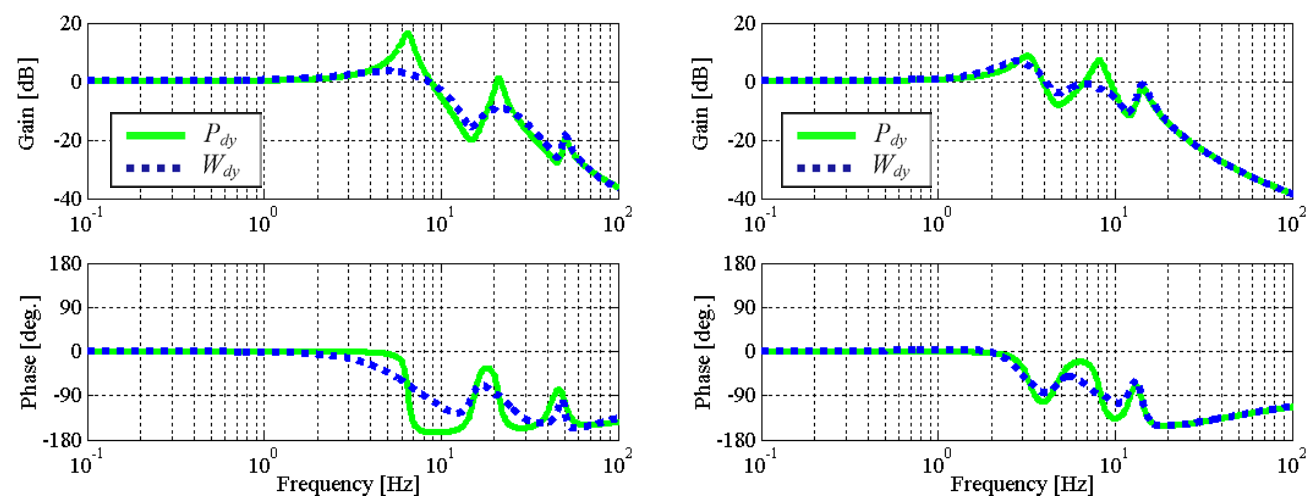

(a) Tube length: $5 \mathrm{~m}$

(b) Tube length: $20 \mathrm{~m}$

Fig. 8 Simulation results for frequency transmissibility characteristics 
These results demonstrate attenuation up to the second resonance frequency and they show that there is no spillover in the higher frequency range. This confirms that the designed control system works effectively.

\subsection{Experimental Results}

We conducted a vibration isolation experiment by mounting the controller designed in $\S 5.1$ on an experimental system. Figure 9 shows the frequency transmissibility characteristics from the floor acceleration to the acceleration of the vibration isolation table for the case of the vibration isolation performance for the acceleration of the floor. Figure 10 shows the time history waveforms of the acceleration of the floor and the acceleration on the vibration isolation table. In order to make the graphs easy to read, a $40-\mathrm{Hz}$ second-order low-pass filter for noise removal was applied to these data and the resulting graphs are shown in Fig.10.

Based on the experimental results for the frequency transmissibility characteristics, when the model matching technique is used, the first and second resonance peaks can be reduced as in the simulation and good control performance can be realized without excitation of higher vibration modes not included in the control model. These results indicate the effectiveness of a controller that uses the model matching technique.

Finally, using the time history waveforms, the root-mean-square values for the floor and table acceleration are found to be $2.71 \times 10^{-3} \mathrm{~m} / \mathrm{s}^{2}$ and $2.65 \times 10^{-4} \mathrm{~m} / \mathrm{s}^{2}$ when $5-\mathrm{m}$ tubes are installed, and $2.99 \times 10^{-3} \mathrm{~m} / \mathrm{s}^{2}$ and $2.41 \times 10^{-4} \mathrm{~m} / \mathrm{s}^{2}$ when $20-\mathrm{m}$ tubes are installed, respectively. Therefore the acceleration of the vibration isolation table is attenuated to about $10 \%$ of the floor vibration. Thus, the effectiveness of this control technique is confirmed.
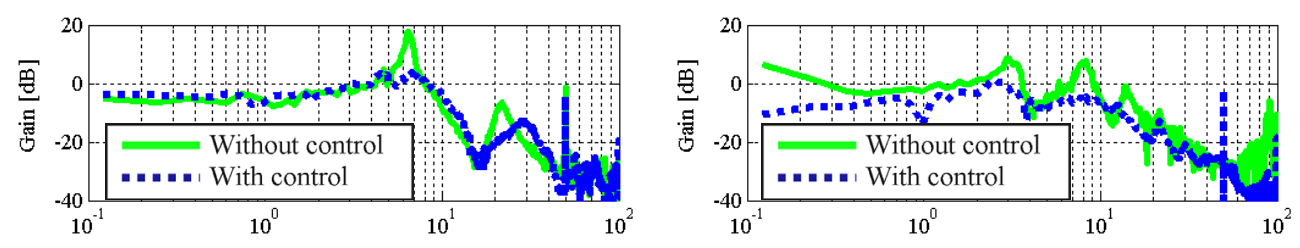

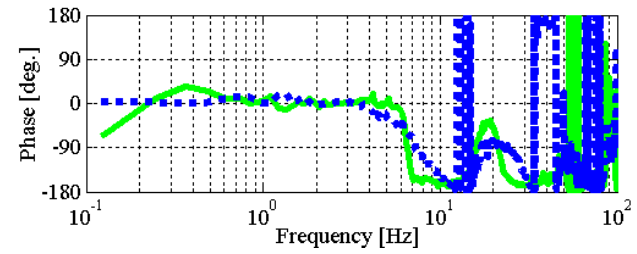

(a) Tube length: $5 \mathrm{~m}$

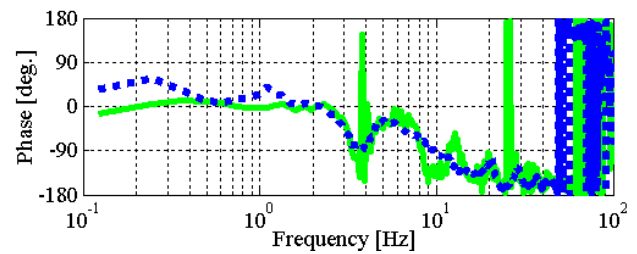

(b) Tube length: $20 \mathrm{~m}$

Fig. 9 Experimental results for frequency transmissibility characteristics

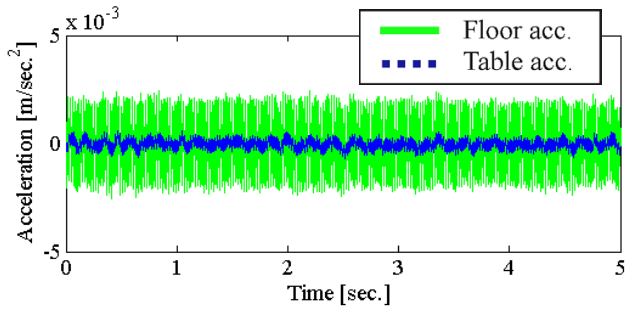

(a) Tube length: $5 \mathrm{~m}$

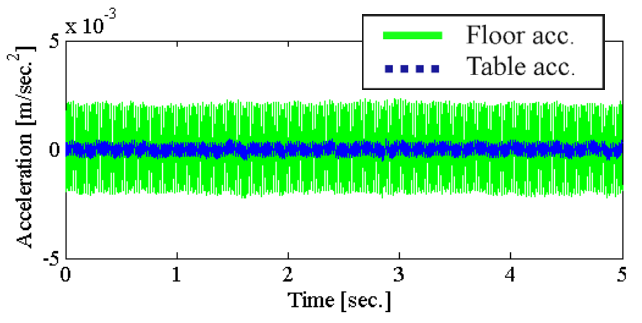

(b) Tube length: $20 \mathrm{~m}$

Fig. 10 Experimental results for time history waveforms 


\section{Conclusions}

The goal of this study was to enhance the performance of an active vibration isolation system having a long tube between the air actuator and the servo valve. A system model was first constructed. Based on this model, we investigated a control system and verified its effect by simulation and experiment. The results of this study can be summarized as follows:

(1) The system characteristics experiment verified that the tube between the air actuator and the servo valve greatly affected the system characteristics.

(2) By incorporating the air in the tube as a virtual mass in the conventional model of a vibration isolation system, we created a model and simulated identification. Consequently, the influence of the air pressure in the tube could be determined.

(3) We designed a controller by applying the model matching technique and conducted a vibration isolation experiment. Even when the air pressure in the tube caused several resonance points to appear, an enhancement in the vibration isolation performance of the system was verified.

\section{Acknowledgement}

We would like to express our gratitude to Dr. Masashi Yasuda at Tokkyokiki Corporation for his valuable advice in regard to this study.

\section{References}

(1) Yasuda M., Osaka T. and Ikeda M., Study of Active Isolation System with Feedforward Control, Transactions of the Japan Society of Mechanical Engineers Series C, Vol.58, No.552 (1992), pp. $2381-2387$ (in Japanese).

(2) Kajiwara K., Hayatsu M., Imaoka S. and Fujita T., Large Scale Active Microvibration Control System Using Piezoelectric Actuators Applied to Semiconductor Manufacturing Equipment, Transactions of the Japan Society of Mechanical Engineers Series C, Vol.63, No.615 (1997), pp. 3735-3742 (in Japanese).

(3) Nishiyama Y., Tagawa Y. and Yasuda M., Control of a 6DOF Microvibration Testing Device Using Air Actuators, Transactions of the Japan Society of Mechanical Engineers Series C, Vol.66, No.643 (2000), pp. 771-777 (in Japanese).

(4) Japan Hydraulics \& Pneumatics Society, Handbook of Hydraulics \& Pneumatics, (1989), pp. 17-47, Ohmsha Ltd. (in Japanese)

(5) J. Jang, Pressure Control of a Volume Terminated Pneumatic Control System with a Long Transmission Line, Journal of the Japan Fluid Power System Society, Vol.34, No.6 (2003), pp. 423-427 (in Japanese).

(6) Tagawa Y. and Fujita T., Design Method for Multiinput Multioutput Microvibration Control System, Transactions of the Japan Society of Mechanical Engineers Series C, Vol.58, No.550 (1992), pp. 1819-1826 (in Japanese).

(7) Tagawa Y., Seino Y. and Yasuda M., Study of 6-DOF Microvibration Control Using Feedforward Control, Transactions of the Japan Society of Mechanical Engineers Series C, Vol.63, No.608 (1997), pp. 1081-1087 (in Japanese). 